\title{
P04-2-3 Poster session
}

\section{Deletion of SIRT1 in the cardiomyocyte exacerbates doxorubicin-induced cardiotoxicity in mice}

\author{
Ryusuke Hosoda, Atsushi Kuno, Yoshiyuki Horio \\ Department of Pharmacology, Sapporo Medical University School of Medicine, Japan
}

Background: SIRT1 is an $\mathrm{NAD}^{+}$-dependent deacetylase that promotes cellular adaptation in response to various stresses by preserving mitochondrial function. Mitochondrial damage plays a key role in pathogenesis of doxorubicin (DOX)induced cardiotoxicity. Here, we examined whether and how SIRT1 in the cardiomyocyte protects against DOX-induced cardiotoxicity.

Methods and Results: Dox (4 IP injections of $5 \mathrm{mg} / \mathrm{kg} /$ week) was administered to mice with cardiomyocyte-specific deletion of SIRT1 by tamoxifen (SIRT1 cKO) and mice without Cre recombinase served as wild type (WT). In Experiment 1, cardiac tissues were sampled 1 week after final DOX injection. Echocardiography at baseline showed that left ventricular (LV) dimension and LV fractional shortening (\%FS, 32.7\% vs. 33.8\%), an index of LV systolic function, were comparable in WT and SIRT1 cKO. In contrast, \%FS after DOX was lower in SIRT1 cKO than in WT (27.3\% vs. 24.6\%, $\mathrm{P}<0.05)$. Cardiac mRNA levels of ANP and BNP, markers of cardiac dysfunction, were higher in SIRT1 cKO than those in WT $(2.4$-fold and 2.4-fold, respectively, $\mathrm{P}<0.05)$. Transcript levels of nuclear-encoded mitochondrial genes (Tfam, Cox5b, Atp5a1, and citrate synthase) were unchanged in the SIRT1 cKO heart. In Experiment 2, both groups of mice received vehicle (PBS) or DOX at the same dose as in Experiment 1, and cardiac tissues were sampled. In DOXtreated mice, myocardial level of nitrotyrosine, a marker of oxidative stress, was significantly higher in SIRT1 cKO by $68 \%$ than WT. The myocardial mitochondrial DNA (mtDNA) copy number determined by qPCR was not affected in SIRT1 cKO compared with WT after DOX. However, level of mtDNA deletion analyzed by a long-range PCR method was significantly increased by DOX in SIRT1 cKO, but such a change was not observed in WT.

Conclusion: Deletion of SIRT1 in the cardiomyocyte exacerbates DOX-induced cardiac dysfunction in mice, probably due to accumulation of deleted mtDNA caused by enhanced oxidative stress, leading to mitochondrial dysfunction. 